Gynäkologische Endokrinologie 2014 · 12:179

DOI 10.1007/s10304-014-0650-2

Online publiziert: 23. August 2014

(c) Springer-Verlag Berlin Heidelberg 2014

Deutsche Menopause Gesellschaft e.V.

Redaktion

Deutsche Menopause Gesellschaft e.V.

P. Stute, Bern

A.O. Mueck, Tübingen

\section{Originalpublikation}

Shufelt CL, Merz CN, Prentice RL et al (2014) Hormone therapy dose, formulation, route of delivery, and risk of cardiovascular events in women: findings from the Women's Health Initiative Observational Study. Menopause 21(3):260-266

\section{Hintergrund}

Eine niedrig dosierte und/oder transdermale Östrogentherapie wird mit einem geringeren kardiovaskulären Erkrankungsrisiko assoziiert. Die Evidenz für diese Aussage ist jedoch begrenzt.

\section{Zusammenfassung der Studie}

Die Women's Health Initiative Observational Study (WHI-OS) ist eine prospektive Kohortenstudie an bei Studienstart 50 bis 79-jährigen postmenopausalen Frauen, die u. a. jährlich per Fragebogen zur Hormontherapie (HT) interviewt wurden. Die HT-Subkategorien waren

- orale niedrig dosierte konjugierte equine Östrogene (CEE; <0,625 mg/ Tag),

- orale CEE in Standarddosis (0,625 mg/Tag),

- orale hoch dosierte CEE (>0,625 mg/ Tag),

- orale Östrogene [CEE + Östradiol $\left.\left(\mathrm{E}_{2}\right)\right]$,

- eine orale Östrogen-Gestagen-Therapie (EPT) mit oralem CEE bzw. $\mathrm{E}_{2}$ plus synthetischem Gestagen bzw. Progesteron und

- eine dosisunabhängige transdermale Östrogentherapie (ET) ggf. plus syn-

P. Stute

Inselspital Bern, Bern

\title{
Einfluss der Östrogendosis und -applikationsform auf das kardiovaskuläre Erkrankungsrisiko
}

thetisches Gestagen bzw. Progesteron bei intaktem Uterus.

Endpunkte dieser Studie waren

1. eine relevante koronare Herzkrankheit (KHK; nichttödlicher Herzinfarkt, Koronartod),

2. ein Apoplex,

3. die kardiovaskuläre Mortalität,

4. alle kardiovaskulären Ereignisse (1., 2. und 3.) und

5. die Gesamtmortalität.

Als Datenbasis dienten etwa 42.000 Frauen, die zum Studienstart eine HT anwendeten (45\% der Gesamtpopulation). Das Follow-up betrug 10,4 Jahre. Der Zeitraum seit der Menopause (< oder $\geq 10$ Jahre) und die HT-Dauer (< oder $\geq 5$ Jahre) wurden als mögliche Einflussfaktoren berücksichtigt. Die meisten Frauen wendeten orale CEE in Standarddosis an. Eine transdermale ET/EPT war - unabhängig von der Zeit seit der Menopause und der HT-Anwendungsdauer - im Vergleich zu oralen CEE in Standarddosis mit einem nichtsignifikant niedrigeren Risiko für eine relevante KHK und einen Apoplex verbunden. Orale niedrig dosierte CEE waren mit einem nichtsignifikant erniedrigten Risiko für alle kardialen Ereignisse, nicht aber für einen Apoplex assoziiert. Beim Vergleich der Östrogentypen zeigte sich ein nichtsignifikant erniedrigtes Apoplexrisiko unter oralem $E_{2}$ im Vergleich zu oralen CEE in Standarddosis. Der Vergleich der oralen EPT mit oralen CEE in Standarddosis zeigte keine Unterschiede in Bezug auf kardio- und zerebrovaskuläre Ereignisse sowie die Mortalität.

Die Autoren kommen zu dem Schluss, dass die Östrogendosis und -applikati- onsform kaum eine Rolle für die kardiovaskuläre Gesundheit spielen, aber orales $E_{2}$ eventuell günstiger bezüglich des Apoplexrisikos sei und eine transdermale HT sowie niedrig dosierte CEE eventuell günstiger bezüglich koronarer Ereignisse.

\section{Kommentar}

> Trotz der Kohortengröße und des langen Beobachtungszeitraums ist eine finale Aussage über das vermutete günstigere kardiovaskuläre Profil einer transdermalen bzw. niedrig dosierten HT nicht möglich.

Dies liegt an den typischen Schwächen von Observationsstudien, der geringen Fallzahl in den Gruppen der transdermalen HT $(n=2187)$ und niedrig dosierten CEE $(n=2149)$ sowie am alleinigen Einschluss von derzeitigen HT-Anwenderinnen bei Studienbeginn. Hierdurch wird die im ersten Anwendungsjahr einer oralen HT erhöhte Inzidenz koronarer Ereignisse nicht miterfasst und möglicherweise die Prävalenz kardiovaskulärer Ereignisse im Beobachtungszeitraum unterschätzt. Und somit erklingt wieder der Ruf nach weiteren Studien ...

\section{Korrespondenzadresse}

PD Dr. P. Stute

Inselspital Bern

Effingerstr. 102, 3010 Bern

Schweiz

petra.stute@insel.ch

Interessenkonflikt. P. Stute gibt an, dass kein Interessenkonflikt besteht. 